\title{
Neuromuscular Junctions Shrink and Expand as Muscle Fiber Size Is Manipulated: in vivo Observations in the Androgen-Sensitive Bulbocavernosus Muscle of Mice
}

\author{
Rita J. Balice-Gordon, ${ }^{1}$ S. Marc. Breedlove, ${ }^{2}$ Steven Bernstein, ${ }^{1}$ and Jeff W. Lichtman ${ }^{1}$ \\ ${ }^{1}$ Department of Anatomy and Neurobiology, Washington University School of Medicine, St. Louis, Missouri 63110, and \\ Department of Psychology, University of California, Berkeley, Berkeley, California 94720
}

Neuromuscular synapses in an androgen-sensitive muscle of sexually mature male mice were repeatedly observed over several-month intervals in normal animals and in animals in which testosterone levels were manipulated. In normal bulbocavernosus muscles, pre- and postsynaptic regions of neuromuscular junctions enlarge as muscle fibers grow. After castration, Junctional area decreased in parallel with muscle fiber atrophy. When testosterone was resupplied to castrated animals, junctions that previously decreased in size then enlarged in parallel with muscle fiber hypertrophy. Surprisingly, these size changes occurred without loss or addition of motor nerve terminal branches or acetylcholine (ACh) receptor regions. Rather, each nerve terminal branch and underlying receptor region became smaller following castration and reenlarged following testosterone treatment.

Several lines of evidence argued that the size changes observed after castration and testosterone treatment were secondary to shrinkage and stretching of the postsynaptic muscle fiber membrane. Following castration, the spaces between synaptic regions decreased in size at the same time and to a similar extent as the regions themselves. Following testosterone replacement, the spaces between synaptic regions expanded and each existing ACh receptor region enlarged. Ultrastructural analysis showed that there was no loss or addition of postsynaptic secondary junctional folds in the muscle fiber membrane (where ACh receptors are located) as junctions shrank and expanded. Rather, folds became more densely packed as muscle fibers atrophied following castration and less densely packed as muscle fibers hypertrophied following testosterone replacement.

From these studies of the bulbocavernosus muscle, as from our previous studies of the sternomastoid muscle, we conclude that neuromuscular junction size is directly coupled to muscle fiber size. Androgens modulate muscle fiber volume directly, leading to a change in the surface area of the muscle fiber membrane, which in turn causes the post-

\footnotetext{
Received Feb. 9, 1990; revised March 30, 1990; accepted April 2, 1990.

We thank Christine Chua for assistance with morphometry and preparation of tissue for ultrastructural analysis, Grady Phillips and Sherman Owens for technica assistance, and Drs. J. Cohen and P. van Mier for helpful discussions and comments on the manuscript. This work was supported by grants from the NIH and MDA to I.W.L., from the NSF and the McKnight Foundation to S.M.B and the Harry Zimmerman Postdoctoral Fellowship of the MDA to R.B.-G.

Correspondence should be addressed to Dr. Rita J. Balice-Gordon, Department of Anatomy and Neurobiology, Washington University School of Medicine, 660 South Euclid Avenue, Box 8108, St. Louis, MO 63110.

Copyright (C) 1990 Society for Neuroscience $0270-6474 / 90 / 082660-12 \$ 03.00 / 0$
}

synaptic specializations to shrink or expand. The concomitant shrinkage and stretching of motor nerve terminals that we observed can only be accounted for by their adhesion to postsynaptic specializations that are also changing size. Thus adhesion, rather than an interchange of diffusible factors, trophic or otherwise, is likely to be the primary determinant of coordinated pre- and postsynaptic enlargement in growing mammalian skeletal muscles.

One reason synaptic connections may remain plastic over long periods of an animal's lifetime is to compensate for changes in the size of postsynaptic cells as animals grow. For example, the same motor nerve terminal must continue to elicit contractions in the same muscle fiber throughout life, even though during that time, the muscle fiber grows tremendously in length and width. A number of studies of neuromuscular junctions in growing animals have shown that motor nerve terminals compensate for the growth of muscle fibers by also growing (see Wernig and Herrera, 1986, for review). It has remained unclear, however, how synapses in general and neuromuscular junctions in particular grow, and what regulates this growth.

A possible strategy for motor nerve terminal growth is that it is regulated by feedback based on muscle fiber activity. For example, as a muscle fiber grows, its junction may become progressively less able to drive the muscle fiber to contract because of a progressively increasing mismatch between the amount of synaptic current and the voltage change obtained in the muscle fiber. As a result of increasingly subthreshold innervation, relatively inactive muscle fibers may respond by releasing or expressing agents that cause motor axons to sprout. An innervating motor axon may add new terminal branches in response to such sprouting stimuli, resulting in an increase in the synaptic efficacy of the enlarging junction, which again reaches the desired suprathreshold level. The muscle fiber would then be restored to its normal level of activity, in turn causing the sprouting stimuli to be down-regulated.

In this way, nerve terminals would add progressively more branches as their target cells grew. Furthermore, when the target muscle fibers reached their full adult size, one might expect that the equilibrium obtained between nerve terminal size and muscle fiber size would be a dynamic steady state (Barker and Ip, 1966; Robbins, 1988). As muscle activity increased, the stimuli for nerve terminal growth might be down-regulated, to the point where terminal branches would be eliminated. This loss would be followed by relative muscle inactivity, causing synapses to add branches. Thus a good deal of remodeling at mature junctions might take place. 


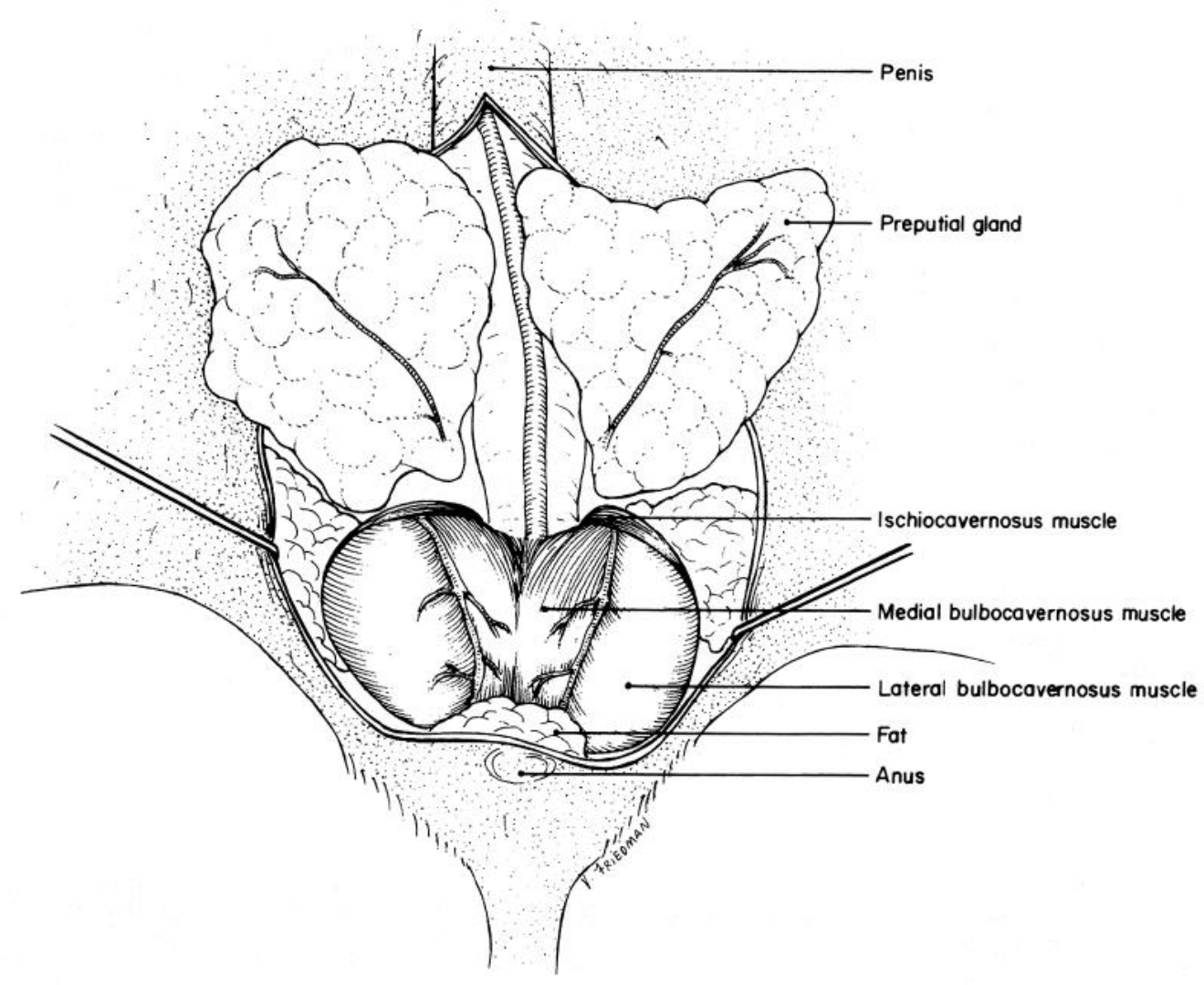

Figure 1. Location of the bulbocavernosus muscles in male mice. Shown is a drawing of the ventral surface of the perineal region. The medial and lateral bulbocavernosus muscles are exposed by midline incision, retraction of the scrotum, and reflection of the overlying preputial glands. Following staining of motor nerve terminals and ACh receptors, a small firepolished glass coverslip on an adjustable lever is gently lowered over the muscle, flattening it slightly and resulting in good optical access to superficial neuromuscular junctions. The dorsal bulbocavernosus muscle (commonly referred to as the levator ani) lies beneath the musculature shown here and is not illustrated.

Observations from several different muscles in both mammals and amphibians support the general scenario described above (see Wernig and Herrera, 1986, and Lichtman, 1988, for reviews). However, directly obtained life histories of the same neuromuscular junctions followed over many months in living mice argued for a different method of neuromuscular synaptic growth (Balice-Gordon and Lichtman, 1990; see also Lichtman et al., 1987). In the sternomastoid muscle, neuromuscular junctions did grow in parallel with muscle fibers, but there was little evidence of addition or retraction of motor nerve terminal branches. The pattern of motor nerve terminals and postsynaptic $\mathrm{ACh}$ receptor regions present at 2 weeks of age enlarged but was otherwise largely unchanged for as long as 1 year. Moreover, the growth of postsynaptic receptor regions was observed to be directly coupled to muscle fiber growth, because existing $\mathrm{ACh}$ receptor regions spread apart as the postsynaptic muscle fiber membrane expanded. These results suggested that adhesion between motor nerve terminals and expanding postsynaptic regions might be the basis for the concomitant increases in junctional and muscle fiber size. The lack of synaptic remodeling observed in the adult sternomastoid muscle supported the idea that neuromuscular synapses, once established, are remarkably stable.

In the study reported here, we pursued these ideas further in several ways. First, we asked whether sternomastoid neuromuscular junctions were exceptional or whether junctions from other muscles also grew by overall expansion without a change in the pattern or number of synaptic regions. Second, to test the idea that muscle fiber size determined junctional size in a passive mechanical way, we studied junctional size in a muscle where muscle fiber size could be both increased and decreased. For example, does muscle fiber atrophy give rise to compaction of neuromuscular junctions without the loss of synaptic regions, or, alternatively, do junctions decrease in size by the loss of synaptic regions? The ability of junctions to increase and decrease in size is of interest because the size of some rodent muscles waxes and wanes seasonally (Forger and Breedlove, 1987), perhaps requiring both up- and down-regulation of synaptic strength. Finally, we studied how these relatively macroscopic changes in junctional size might be manifested at the ultrastructural level. In particular, we were interested in whether junctional size changes lead to changes in the number of postsynaptic secondary junctional folds (where ACh receptors are located), or whether junctional fold density alone is altered as junctions change size.

With these aims in mind, we studied neuromuscular junctions in the bulbocavernosus muscle of sexually mature male mice as muscle fiber size was reversibly altered. The bulbocavernosus muscle (see Fig. 1) is one of a complex of androgen-sensitive muscles which wraps around the base of the penis and is involved in copulatory behaviors (Sachs, 1982; Hart and Melese d'Hospital, 1983). This muscle complex has been widely used 
for the study of hormonal effects on muscles, motor neurons, and neuromuscular junctions (Bleisch and Harrelson, 1989; see Breedlove, 1986, for review).

Muscle fiber size in the bulbocavernosus muscle can be reversibly manipulated with androgens; removal of testosterone by castration results in a reduction of muscle mass (Wainman and Shipounoff, 1941) without a change in muscle fiber number (Venable, 1966a). When testosterone is resupplied, muscle mass is restored to normal levels (Wainman and Shipounoff, 1941) as atrophied fibers enlarge without addition of new fibers (Venable, 1966a). Androgens exert these effects by acting directly on muscle fibers (Powers and Florini, 1975; Rand and Breedlove, 1987). Because neuromuscular synapses in the bulbocavernosus muscle are accessible to direct observations in living animals, we could repeatedly study the same neuromuscular junctions as muscle fiber size was altered with androgens. A portion of these results has been reported in abstract form (Breedlove et al., 1988).

\section{Materials and Methods}

Three-month-old CF1-B male mice (Harlan/Sprague-Dawley) were used for the present experiments. Methods for anesthesia, preparation of animals, repeated in vivo imaging of motor nerve terminals and $\mathrm{ACh}$ receptors, and relevant controls are described fully elsewhere (Lichtman et al., 1987; Balice-Gordon and Lichtman, 1990). Only methods specific to the present study are included here.

Repeated observations of neuromuscular junctions and manipulation of muscle fiber size. The medial and lateral bulbocavernosus muscles were exposed with a midline incision in the scrotum and reflection of the overlying preputial glands (Fig. 1; see also Fig. 3). Following staining of motor nerve terminals with a $10 \mu \mathrm{M}$ solution of 4-Di-2-ASP (Molecular Probes, Eugene, OR; Magrassi et al., 1987) and of postsynaptic ACh receptors with a nonsaturating dose of rhodaminc-conjugated $\alpha$-bungarotoxin (Rich and Lichtman, 1989a), the muscle was covered with a small, fire-polished, glass coverslip and superficial neuromuscular junctions imaged as described in Lichtman et al. (1987).

In one group of mice, the normal pattern of growth of bulbocavernosus neuromuscular junctions was determined by making repeated observations of the same junctions at 1 month intervals for 2-6 months. In another group of mice, after the initial observation, the animals were castrated via a midline abdominal incision. One month later, a second observation of the same junctions was made. Following the second observation, a small silastic capsule $(10 \mathrm{~mm}$ long, $1.6 \mathrm{~mm}$ inner diameter, $3.2 \mathrm{~mm}$ outer diameter) filled with crystalline testosterone (Steraloids, Wilton, NH) constructed as described in Smith et al. (1977) was implanted beneath the skin of the neck. Implants such as these have been shown to produce scrum levels of testosterone in the physiological range of $1 \mathrm{ng} / \mathrm{ml}$ for several months in male rodents (Smith et al., 1977; Menniti and Baum, 1981). However, in distinction to normal animals, there is no feedback inhibition of this release; therefore, in some animals the effective dose of testosterone was probably greater than normal (see for example, Fig. 3, last panel). One month after the capsule was implanted, a third and final observation of the same junctions was made.

To study how ACh receptors were redistributed as junctions changed size, we followed the fate of existing and newly inserted receptors as muscle fiber size was manipulated (Balice-Gordon and Lichtman, 1990). Briefly, receptors in junctions from normal muscles were labeled with a nonsaturating dose of rhodamine $\alpha$-bungarotoxin. One month after castration, those original labeled receptors remaining at the same junctions were rephotographed. Additional $\alpha$-bungarotoxin was then applicd to label receptors inserted since the first view and the junctions were photographed again. One month after testosterone replacement, ACh receptors remaining from the previous view were photographed. AMCA $\alpha$-bungarotoxin (which fluoresces blue; Balice-Gordon and Lichtman, 1990) was then applied to label new receptors inserted since the previous view and the junctions were photographed again.

Two additional experiments were performed. In 1 set of animals, following castration, additional observations were made at 1 month intervals for a further 3-6 months without testosterone replacement. In another set of animals, following castration, testosterone replacement was continued and additional observations were made at 1 month intervals for a further 3-6 months.

Determination of neuromuscular junctional size and of muscle fiber size. Junctional area, length, and width were determined using a digital bitpad attached to a computer. Junctional area was measured by drawing the smallest smonth perimeter around a digitally stored image of each junction so that all branches were included. Junctional length was measured along a line parallel to the long axis of the muscle fiber. Junctional width was measured along a line perpendicular to that for junctional length at the widest part of the junction.

Muscle fiber diameter in normal and experimental mice was determined by staining frozen sections of bulbocavernosus muscles for myofibrillar ATPase (Guth and Samaha, 1970) and then tracing them with a camera lucida onto the bitpad. At least 100 muscle fibers from each of 5 muscles were measured for each age or experimental group. Because of the complex configuration of muscle fiber origin and insertion in the bulbocavernosus, it was not possible to accurately measure muscle fiber length.

Ultrastructural analysis of postsynaptic specializations. To study the effect of changing muscle fiber size on the number and density of secondary junctional folds, muscles were prepared for transmission electron microscopy in the conventional manner following staining of superficial junctions for acetylcholinesterase (Karnovsky and Roots, 1964). The density of junctional folds was determined from electron photomicrographs by counting the number of folds per micron in at least 3 regions of each junction.

We estimated the amount of nonspecific shrinkage of normal, castrated, and testosterone-treated muscles prepared in this fashion in 2 ways. First, we compared myofibrillar sarcomere length from longitudinally sectioned muscles in the electron microscope with apparent sarcomere length (the distance between A-bands) from in vivo preparations. Second, we compared the average width of primary synaptic gutters from electron photomicrographs with the average width of postsynaptic ACh receptor areas from junctions viewed in the light microscope. Using these criteria, we estimated that muscles prepared for transmission electron microscopy shrank on average approximately 5$10 \%$ and that the degree of shrinkage was similar in normal, castrated, and testosterone-treated muscles. Our analysis of the relative density of postsynaptic secondary junctional folds in muscles prepared for transmission electron microscopy is thus unlikely to be affected by nonspecific muscle shrinkage.

To examine the total number and density of secondary synaptic folds, muscles were also prepared for scanning electron microscopy following acid digestion (Desaki and Uehara, 1981). We found that our results were most consistent if we modified the protocol of Desaki and Uehara (1981) as follows. After fixation, the osmication steps were omitted. The muscle was teased into strips $1-2 \mathrm{~mm}$ wide which were affixed onto glass slides with a thin layer of epoxy. Tissue on slides was then immersed in $8 \mathrm{~N} \mathrm{HCl}$ at $60^{\circ} \mathrm{C}$ for $20-30 \mathrm{~min}$ and then was vigorously sprayed for an additional 10-15 min with a small-caliber needle at tached to a syringe filled with hot $\mathrm{HCl}$. This $\mathrm{HCl}$ treatment resulted in muscle fibers separated from each other and stripped of connective tissue, on which postsynaptic specializations and occasional nerve terminals were clearly visible.

After the muscle pieces were rinsed in water, the tissue was dehydrated, critical-point-dried, mounted on stubs, and sputter-coated with gold. The density of secondary junctional folds was measured from photomicrographs by counting the number of folds per linear micron along the long axis of primary synaptic gutters which were entirely en face. The folds in gutters from at least 3 regions per junction were counted.

The degree of nonspecific shrinkage (estimated as described above) was more pronounced in tissue prepared for scanning electron microscopy than for transmission electron microscopy, probably as a result of critical point drying. Furthermore, the degree of this nonspecific shrinkage varied between normal, castrated, and testosterone-treated muscles. Junctional area was $4,5.4$, and 3.4 times less, respectively, than that determined from muscles in living animals (Table 1). To obtain an estimate of junctional fold number based on a linear dimension rather than on area, we multiplied the linear correlate of these area numbers (the square root of $4,5.4$, and 3.4) by the raw number of folds per linear micron measured from junctions from each group of muscles. However, even the differences in the unnormalized data were statistically significant (see Table 1), meaning that this normalization did not affect the qualitative conclusion reached. 


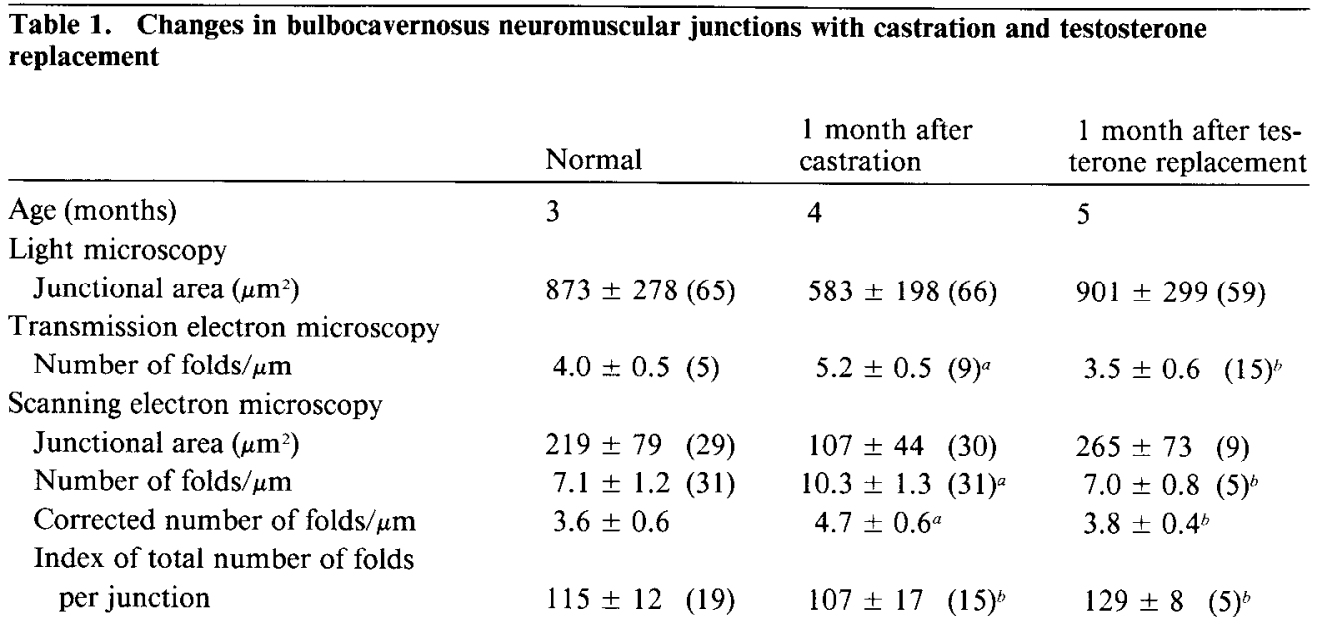

All values expressed as average \pm standard deviation. The number of measurements appears in parentheses.

a Significantly different from normal values; $p<0.001$ (Student's $t$-test).

${ }^{b}$ Not significantly different from normal values (Student's $t$-test).

All values are expressed as averages \pm standard deviation. The number of observations or measurements is shown in parentheses.

\section{Results}

Bulbocavernosus neuromuscular junctions grow without remodeling

Before assessing how junctions change when muscle fiber size is experimentally manipulated with androgens, it was necessary to know what happens to junctions over similar intervals in normal animals. Furthermore, this allowed us to determine if results obtained in the sternomastoid muscle (Lichtman et al., 1987; Balice-Gordon and Lichtman, 1990) were typical of growth-related changes in other muscles. We studied how neuromuscular junctions grow in the bulbocavernosus muscle of male mice ( 12 animals) by repeatedly visualizing both motor nerve terminals and postsynaptic $\mathrm{ACh}$ receptor regions of the same junctions $(n=48)$ at 1 month intervals from 3 to 6 months of age (42 junctions viewed at 3 different times; 6 junctions viewed 4 times). Each junction chosen for study at the first observation was successfully relocated, although occasionally junctions were displaced on their sides, partially obscuring them. In these cases, only regions of the junction that remained visible were compared from view to view.

In each junction, there was overall growth of the motor nerve terminal and $\mathrm{ACh}$ receptor distribution over the interval studied (Fig. 2). Comparison of the area of the same junctions at 3 months and 5 months of age showed that every junction increased in area between 13 and $38 \%$ (by an average of $27 \pm 9 \%$ (42) [average $\pm \mathrm{SD}(N)$ ]). The average junctional area was 921 $\pm 272 \mu \mathrm{m}^{2}$ at 3 months of age and $1170 \pm 246 \mu \mathrm{m}^{2}$ at 5 months of age. In more than $90 \%$ of the junctions we studied ( 44 of 48 ), there was no evidence of ongoing addition or retraction of nerve terminal branches or receptor regions as growth occurred. Rather, there was overall enlargement of existing regions which resulted in an expanded but otherwise unchanged pattern of preand postsynaptic regions (Fig. 2). As this enlargement occurred, a precise alignment was maintained between motor nerve terminal branches and postsynaptic $\mathrm{ACh}$ receptor regions.

In 3 junctions, a small synaptic region was observed to be added over the interval studied. In 1 junction, a small region was observed to be lost. In each of these cases, the altered regions were quite small, less than $10 \mu \mathrm{m}$ in length, representing 3-5\% of the total motor nerve terminal branch length of these junctions. These relatively minor alterations had no significant effect on overall junctional growth because the junctions that added or lost a region grew to the same extent as the junctions that did not.

Junctional growth occurred in parallel with muscle fiber growth. For example, junctional width increased in every junction between 10 and $27 \%$, by an average of $18 \pm 4 \%$ (42). The average junctional width was $28 \pm 8 \mu \mathrm{m}$ at 3 months and $33 \pm 8 \mu \mathrm{m}$ at 5 months of age. Muscle fiber diameter similarly increased on average $20 \%$ during the same interval, from $40 \pm 7 \mu \mathrm{m}$ to $48 \pm 6 \mu \mathrm{m}$.

To study how $\mathrm{ACh}$ receptors were displaced as junctions and muscle fibers enlarged, we followed the fate of originally labeled $\mathrm{ACh}$ receptors in the muscle fiber membrane as growth occurred. Existing receptor regions were observed to spread apart in the postsynaptic membrane and define a larger postsynaptic area as muscle fibers enlarged. New receptors were then observed to be intercalated throughout the enlarged junctional region. These results are the same as those previously described in the growing mouse sternomastoid muscle (Balice-Gordon and Lichtman, 1990).

\section{Neuromuscular junctions shrink after castration and expand after testosterone treatment}

By manipulating androgen levels we studied how the size and pattern of nerve terminals and receptor regions of bulbocavernosus neuromuscular junctions would be affected by changes in muscle fiber size. Junctions were viewed first in 3-month-old male mice which were then castrated to reduce testosterone levels. The same junctions were revisualized 1 month later. Following the second view, testosterone was resupplied to the animals using a subcutaneously implanted silastic capsule filled with testosterone. Although serum levels of testosterone were not measured in this study, the effect of testosterone delivery was monitored by measuring muscle and seminal vesicle wet 


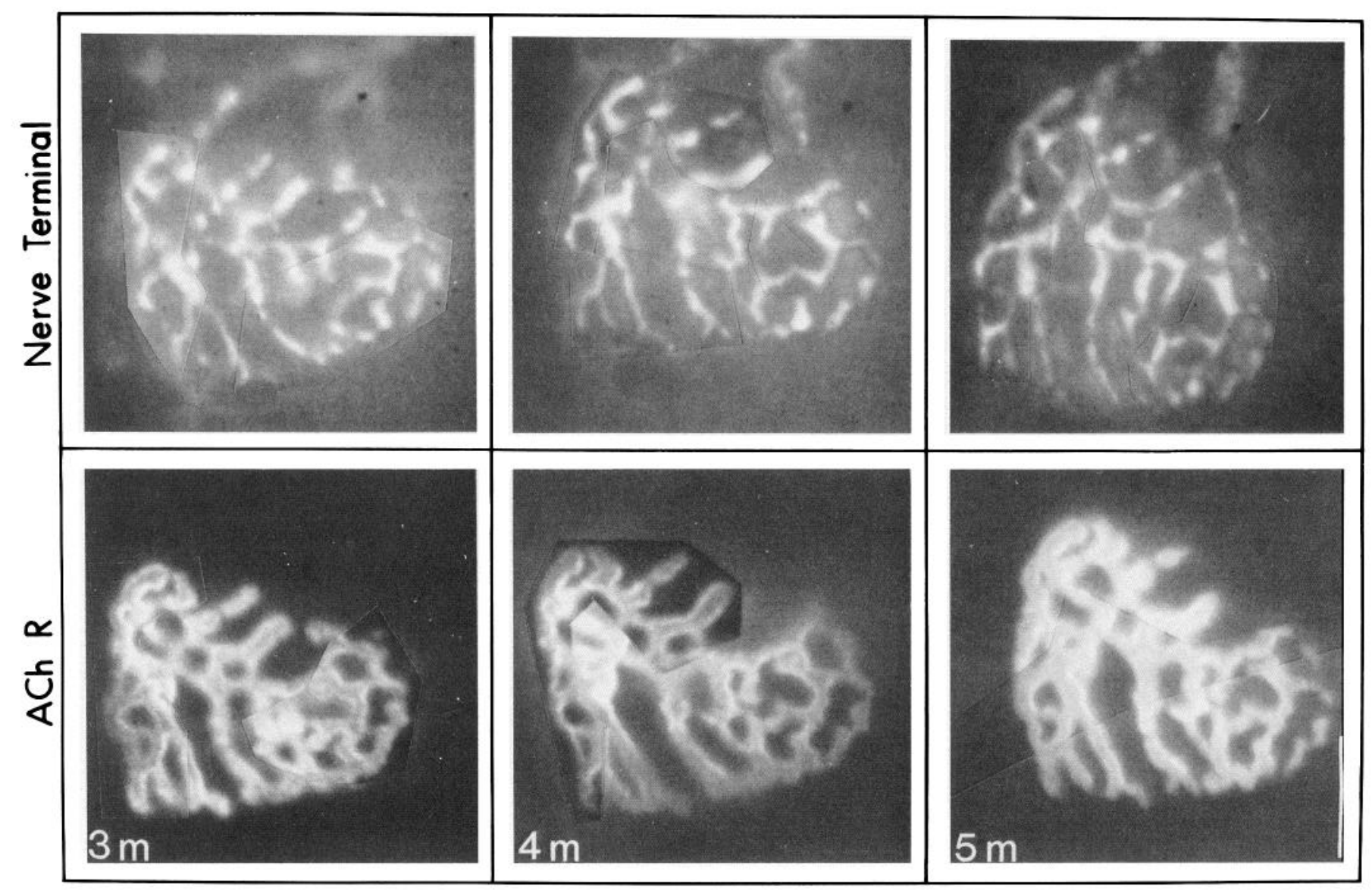

Figure 2. Bulbocavernosus neuromuscular junctions grow without remodeling. Top, A 4-Di-2-ASP-stained motor nerve terminal from a 3-monthold mouse which was subsequently restained and reviewed at 4 and 5 months of age. The preterminal axon can be seen at the top of each panel, where it bifurcates before giving rise to the terminal branches of this junction. Bottom, Underlying ACh receptors stained with a nonblocking dose of rhodamine $\alpha$-bungarotoxin. The predominant mode of growth appears to be enlargement of existing synaptic regions without much evidence of ongoing addition (or loss) of motor nerve terminal branches or ACh receptor areas. As muscle fibers grow between views, existing labeled receptors are observed spread apart in the postsynaptic membrane, defining a larger postsynaptic area. When newly inserted receptors are labeled with a second color of $\alpha$-bungarotoxin, they are observed to be intercalated throughout the enlarged junction and not preferentially at the ends or edges of existing receptor regions (not shown for this junction; see Balice-Gordon and Lichtman, 1990). A precise alignment is maintained between motor nerve terminals and receptor regions as junctions grow, suggesting that pre- and postsynaptic elements of junctions enlarge simultaneously. Because junctions were observed after the period of rapid growth of mice, junctional enlargement is less pronounced than at earlier ages. Junctional area (determined from receptor staining) at 3 months, $1497 \mu \mathrm{m}^{2}$; at 4 months, $1564 \mu \mathrm{m}^{2}$, and at 5 months, $1625 \mu \mathrm{m}^{2}$. In this and all subsequent figures, muscle fibers are oriented vertically and the age of the animal at each view is shown in the lower left-hand corner of the photograph. Because junctions were often in multiple focal planes, we either made photomontages or used an interactive software program written by L. Hedayati and J. Voyvodic in the IMAGR language which enabled us to splice together parts of images. Scale bar, $20 \mu \mathrm{m}$.

weight, both of which approached normal levels after 3-4 weeks in a control group of 5 animals which received testosterone after 1 month of castration.

The effect of castration and subsequent testosterone replacement on the bulbocavernosus muscle from one mouse is shown in Figure 3. One month after castration, visual inspection of the muscle and surrounding structures showed that they had decreased in size. Five animals were killed at this point and muscle fiber cross-sectional area measured; this decreased on average $51 \%$ over normal muscles, from $1340 \pm 173 \mu \mathrm{m}^{2}$ in normal animals at 4 months of age to $663 \pm 178 \mu \mathrm{m}^{2} 1$ month after castration. When testosterone was resupplied for approximately 1 month, atrophied muscles were observed to reenlarge. In another group of 6 animals, measurement of muscle fiber crosssectional area showed that muscle fibers had regained their normal size, increasing to $1281 \pm 221 \mu \mathrm{m}^{2}$.

A total of 130 junctions ( 26 mice) were repeatedly viewed at
3 different time points as muscle fiber size was reversibly manipulated in this fashion. One month after castration, every junction decreased in area between 18 and $54 \%$, on average 33 $\pm 12 \%$ (66; Fig. 4). In $95 \%$ (123 of 130 ) of the junctions studied, each of the regions present at the first observation was present but was smaller at the second view. In $5 \%$ of the junctions ( 7 of 130), 1 or 2 small junction regions less than $10 \mu \mathrm{m}$ in length were lost following castration.

The decrease in junctional size was also evident in the distance between nearby nerve terminal branches, which decreased in size to a similar extent as the branches themselves (Fig. 4). This argues that motor nerve terminals did not become smalier by retracting at the ends of each branch, because if this were the case one would expect that the spaces between branches, if anything, would get bigger rather than smaller (see bars in Fig. 4). Similarly, postsynaptic ACh receptor regions became smaller and the spaces between regions became smaller as well. This 


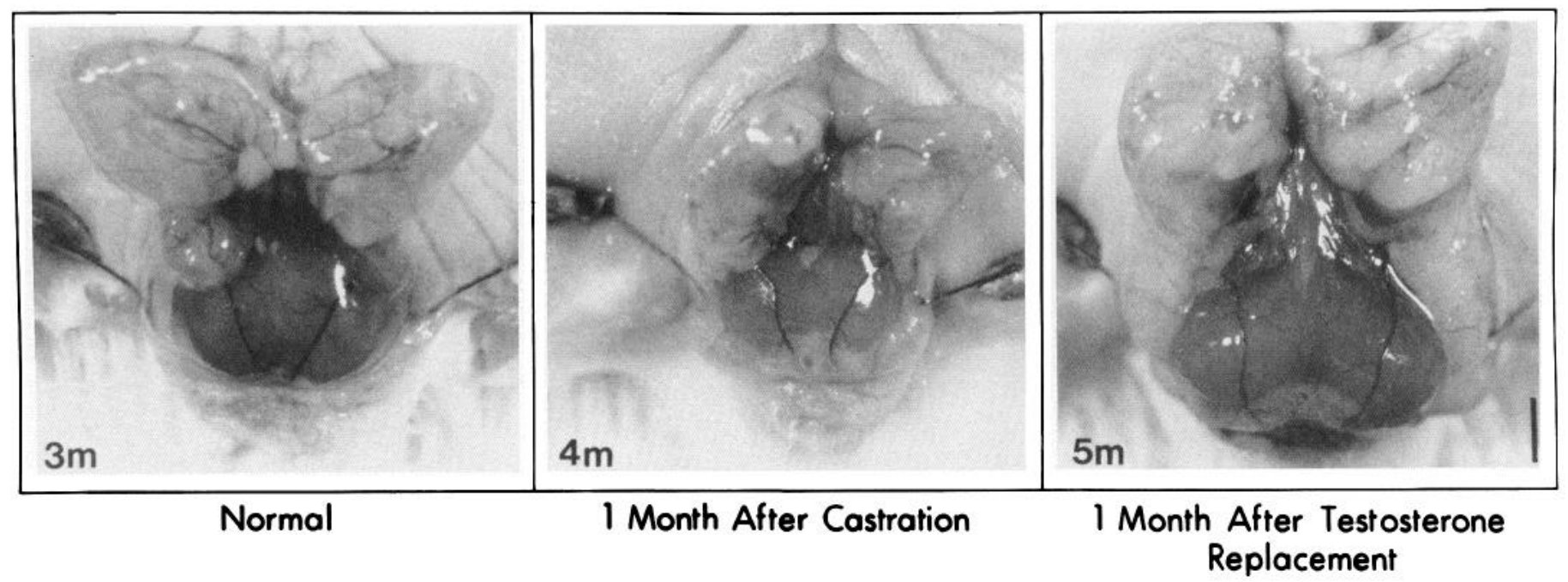

Figure 3. Effect of castration and subsequent testosterone replacement on bulbocavernosus muscles followed in one mouse. Left, Bulbocavernosus muscles from a 3-month-old mouse. After the photograph was taken, the testes were removed. Middle, One month later, the same muscles were photographed again. At this time point, muscles such as this one decreased in weight and muscle fiber cross-sectional area 30-50\%. A capsule of testosterone was then implanted subcutaneously. Right, One month later, the same muscle was again photographed. Following testosterone replacement, muscles generally enlarged to near normal sizes; in this example, the muscle hypertrophied beyond its original size (see Materials and Methods). Scale bar, $3 \mathrm{~mm}$.

result shows that receptor regions do not become smaller by receptors being sculpted away from the edges of each region. In every junction, as synaptic regions became smaller, motor nerve terminals and receptor regions remained aligned, suggesting that they decrease in size at the same time and to the same extent (Fig. 4).

Another potential explanation for the decrease in junctional size following castration is that junctions buckled or wrinkled as muscle fibers atrophied. To evaluate this possibility, we followed an additional 17 junctions that appeared in profile rather than en face before and after castration. If junctions decreased in size by buckling or infolding, one would expect to see evidence of this in the way junctions protruded from the surface of muscle fibers or intruded into their surface. However, none of the junctions in profile on atrophied muscle fibers either collapsed into the muscle fiber or showed signs of protruding. Taken together, these observations suggest that the decrease in junctional size observed after castration appears most consistent with overall shrinkage both pre- and postsynaptically.

After the second observation was made, testosterone replacement was begun and junctions were revisualized 1 month later. Motor nerve terminal branches and postsynaptic ACh receptor regions were observed to reenlarge to near normal sizes (Table 1). Ninety-six percent of the junctions followed (125 of 130) enlarged exclusively by reexpansion of existing synaptic regions. In these junctions, no new synaptic regions were observed to be added during testosterone replacement, as might have been expected, for example, if motor neurons compensate for muscle fiber hypertrophy by adding new terminal branches. In 5 junctions, small regions (less than $10 \mu \mathrm{m}$ in length) were added in addition to junctional expansion. However, even in these few cases, the size and pattern of the junctions were quite similar to those seen at the first view.

Despite enlargement of each pre- and postsynaptic region, the spaces between synaptic regions also increased in size (see bars in Fig. 4). This observation suggested that the entire junction and surrounding muscle fiber membrane expanded as muscle fibers hypertrophied following testosterone treatment.

\section{The branching pattern of neuromuscular junctions remains largely stable following prolonged castration or prolonged testosterone treatment}

Overall junctional shrinkage without substantial loss of synaptic regions was also evident when muscle fibers were allowed to atrophy for more than 1 month following castration. Following prolonged castration ( 7 animals, 21 junctions studied for a total of 5 months), junctions decreased in size (Fig. 5) in parallel with muscle fiber atrophy. Four months after castration, each junction was between 28 and $59 \%$ of its original area (on average $44 \pm 5 \%$ ) and was on average $30 \%$ of the area of junctions from age-matched normal muscles). After this time, junctional size and muscle fiber size changed very little, if at all, over the next 1-2 months. At 15 of 21 junctions, this loss of junctional area occurred without loss of synaptic regions. However, at 6 of 21 junctions in chronically castrated animals, loss of small regions was observed at long time points (Fig. 5, last panel). Although this may indicate that occasionally some synaptic regions are lost as muscle fibers atrophy, the length of lost regions was in each case quite small when compared to the decrease in junctional size.

After castration (for 1 month) followed by prolonged testosterone replacement, junctions continued to hypertrophy in parallel with muscle fibers ( 5 animals, 17 junctions studied for a total of 5 months after testosterone replacement). Four months after testosterone replacement, each junction increased in area between 21 and $57 \%$ (on average $39 \pm 7 \%$ over their size 5 months earlier) and were $11 \%$ larger on average than junctions from age-matched normal muscles. Most of these junctions (12 of 17) continued to enlarge by expansion without a change in the pattern of motor nerve terminal branches or ACh receptor regions. At 5 junctions, addition of 1 or 2 small synaptic regions was observed at long time points. However, the extent and 


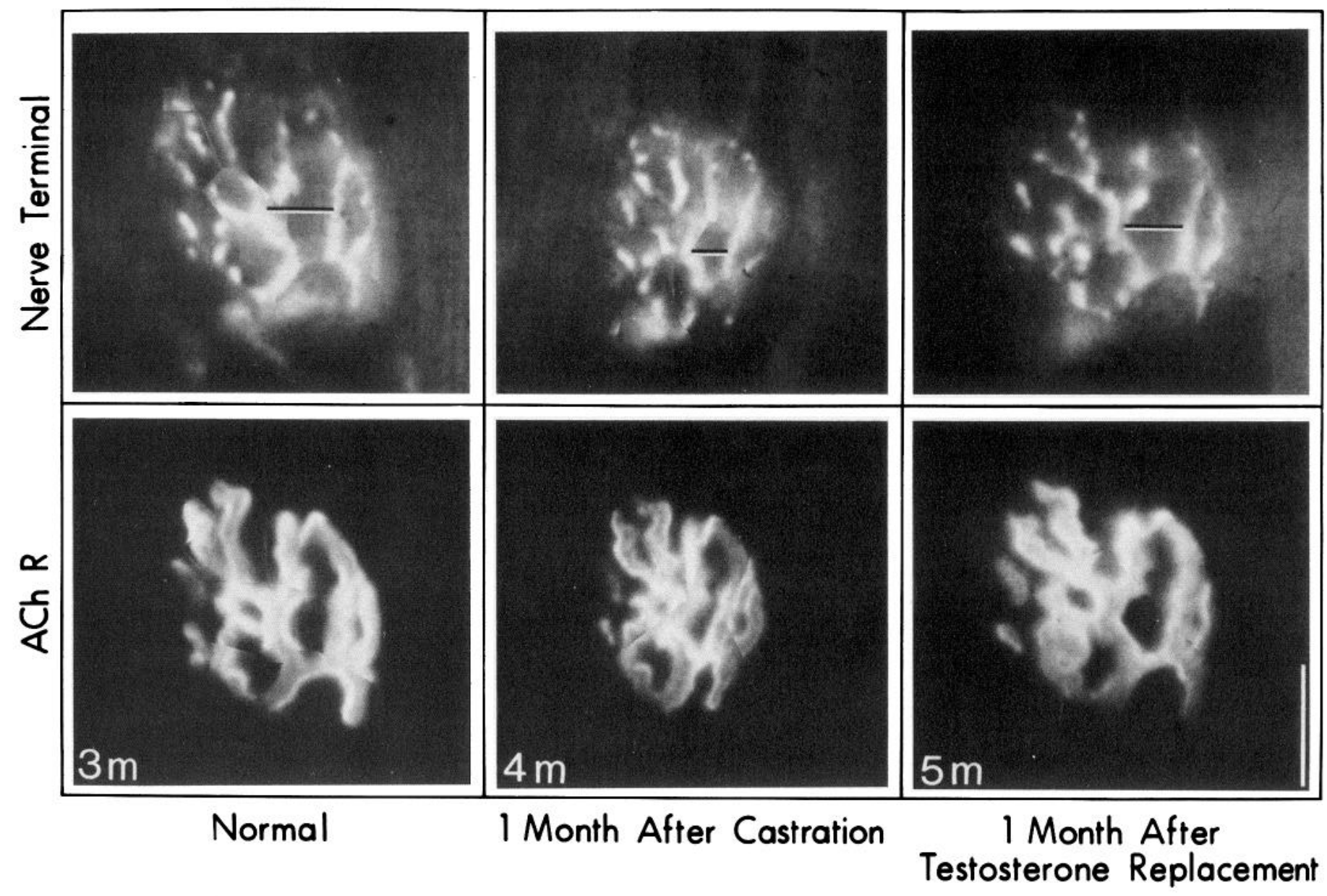

Figure 4. Bulbocavernosus neuromuscular junctions shrink after castration and expand after testosterone treatment without remodeling. Left, Motor nerve terminals stained with 4-Di-2-ASP (top) and underlying ACh receptors labeled with rhodamine $\alpha$-bungarotoxin (bottom) from a neuromuscular junction from a normal 3-month-old mouse. Immediately after these photographs were taken, the animal was castrated. Middle, One month later, the same junction was restained and photographed. Junctional size decreased in proportion to a decrease in muscle fiber surface area. The pattern and number of nerve terminal branches and postsynaptic receptor regions are similar in each view. Each nerve terminal branch and receptor area decreased in size, as did the spaces between synaptic regions (bar), leading to an overall compaction of the junction. Following the second observation, testosterone was resupplied to the animal. Right, One month later, the same junction was again photographed. Junctional size increased in proportion to an increase in muscle fiber size. Each synaptic region and the spaces between regions (bar) enlarged without a change in the number of synaptic regions. Presynaptic motor nerve terminals and underlying ACh receptors remained precisely aligned as these size changes occurred. Junctional area at first view, $752 \mu \mathrm{m}^{2}$; after castration, $480 \mu \mathrm{m}^{2}$; after testosterone treatment, $771 \mu \mathrm{m}^{2}$. Changes in junctional area for normal, castrated, and testosterone-treated muscles are summarized in Table 1 . Scale bar, $20 \mu \mathrm{m}$.

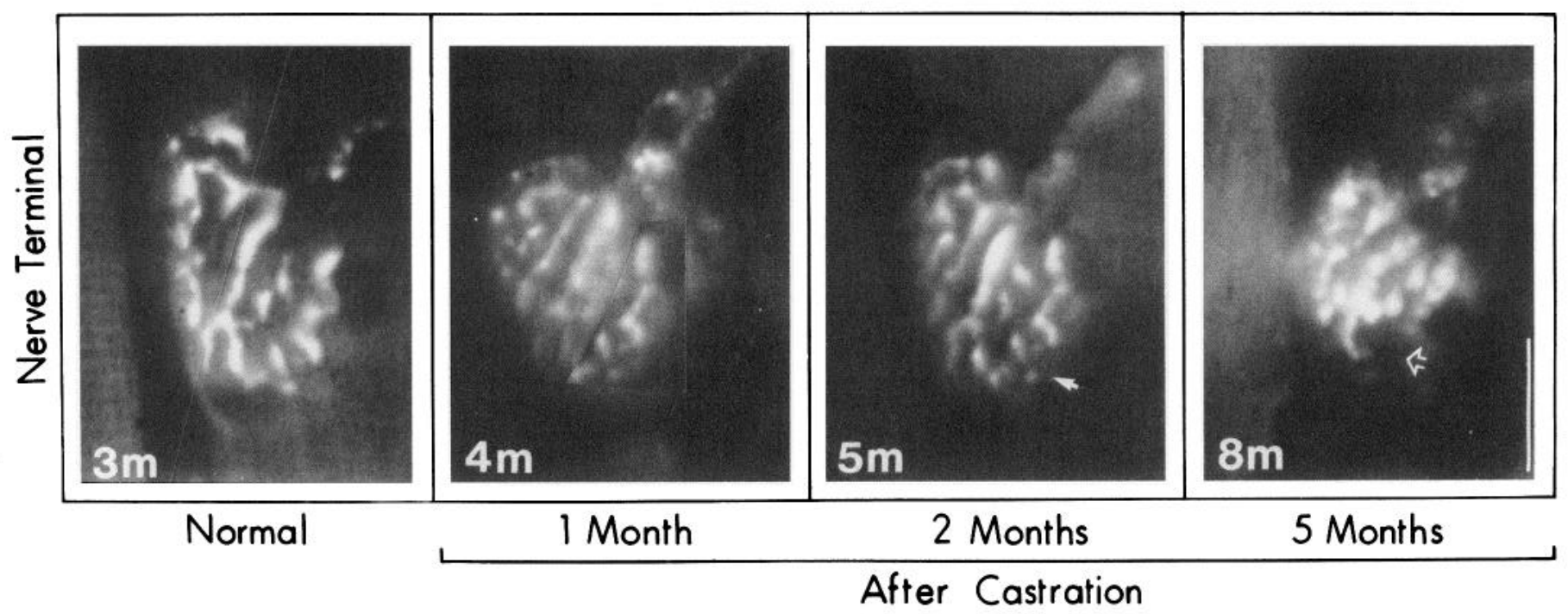



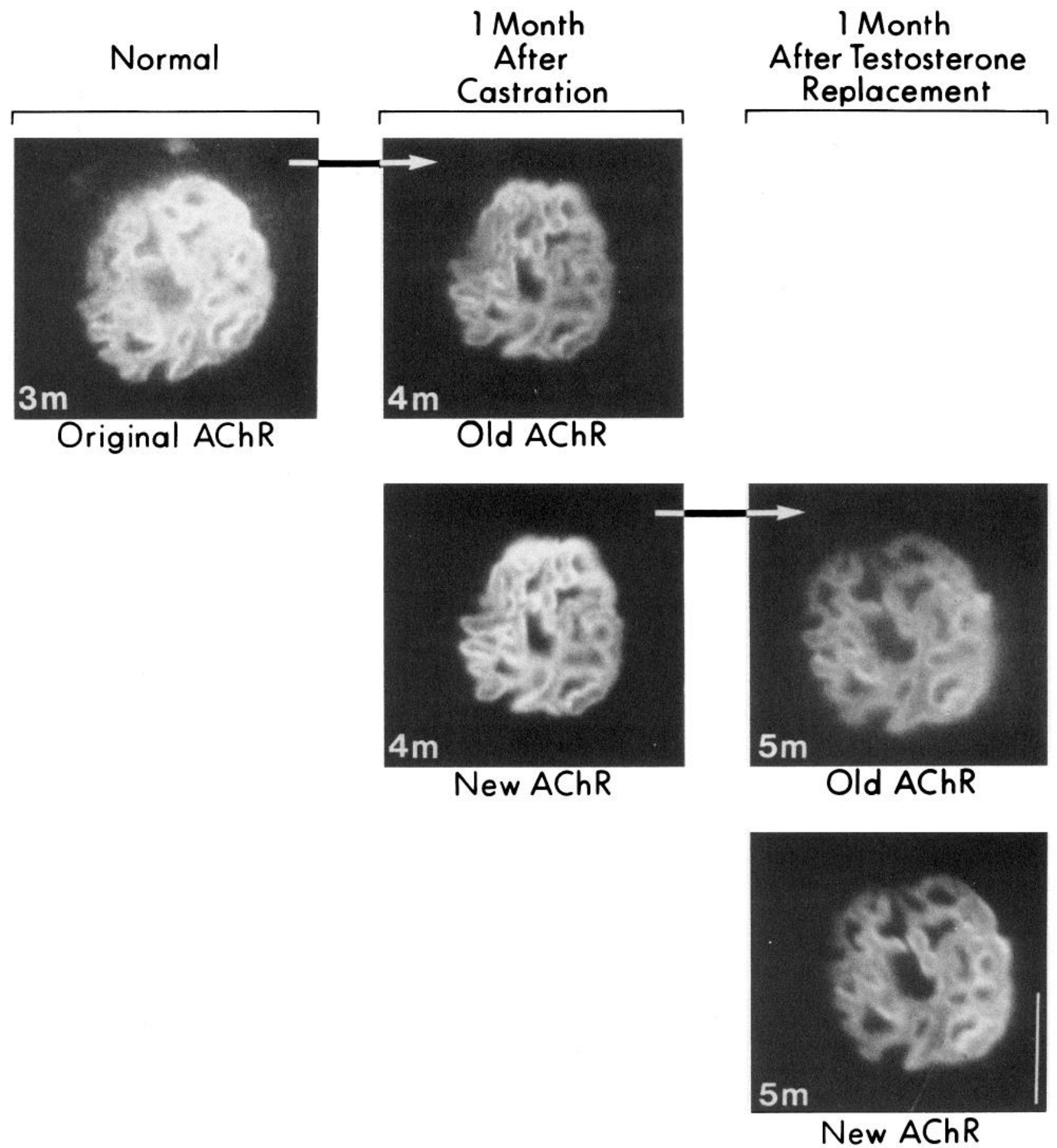

Figure 6. Existing ACh receptors are redistributed in the muscle fiber membrane as muscle fiber size is altered with androgens. Left, Postsynaptic ACh receptors labeled with rhodamine conjugated $\alpha$-bungarotoxin from a junction from a 3-month-old mouse (original AChR). The mouse was subsequently castrated. Middle, Original $\alpha$-bungarotoxin labeled receptors remaining 1 month after castration (old AChR) define a smaller postsynaptic area. The spaces between receptor-rich regions have also decreased in size. Receptors inserted since the previous observation (new AChR) were labeled with additional $\alpha$-bungarotoxin to enable the experiment to be repeated 1 month after testosterone treatment. Right, Following testosterone replacement, existing receptors (old AChR) spread apart as muscle fibers hypertrophy, defining a larger postsynaptic region. New receptors (labeled with a second color of $\alpha$-bungarotoxin; see Materials and Methods) are intercalated throughout the enlarged postsynaptic region and not preferentially at the ends or edges of existing receptor regions. Original receptor area at first view, $748 \mu \mathrm{m}^{2}$; new receptor area 1 month after castration, $597 \mu \mathrm{m}^{2}$; new receptor area 1 month after testosterone replacement, $770 \mu \mathrm{m}^{2}$. Scale bar, $20 \mu \mathrm{m}$.

Figure 5. Remodeling of junctional regions following prolonged castration. Shown are 4 views of a 4-Di-2-ASP-stained nerve terminal arborization followed for 5 months after castration. Until 2 months after castration, the principal change in the junction is one of shrinkage. At 5 months after castration (animal 8 months old), the motor nerve terminal branches marked with an arrow have been lost. Junctional area at first view, $527 \mu \mathrm{m}^{2}$; 1 month after castration, $415 \mu \mathrm{m}^{2} ; 2$ months after castration, $386 \mu \mathrm{m}^{2} ; 5$ months after castration, $302 \mu \mathrm{m}^{2}$. Scale bar, $20 \mu \mathrm{m}$. 


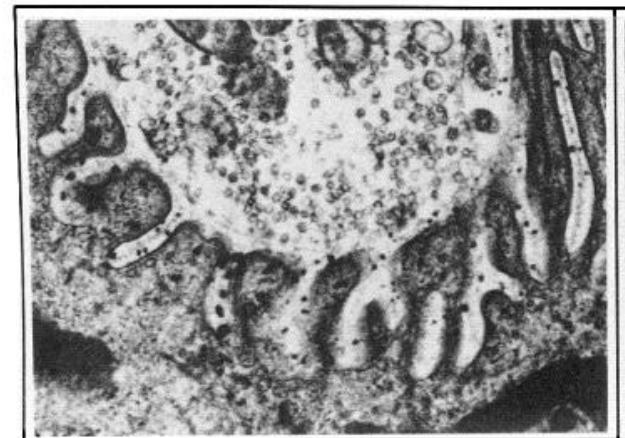

Normal

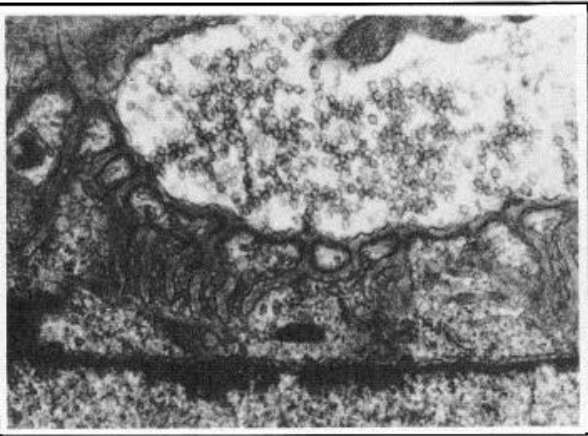

IMonth After Castration

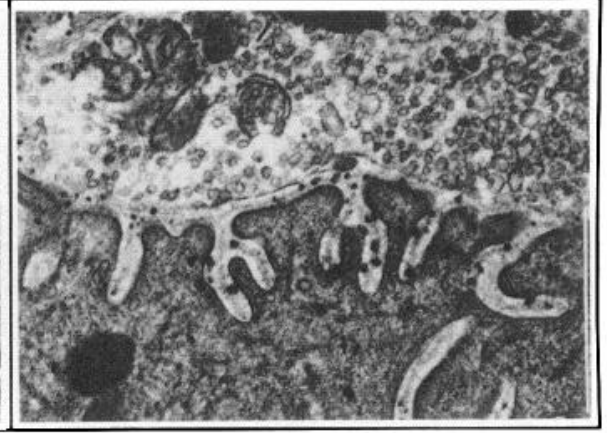

I Month After Testosterone Replacement

Figure 7. Density of postsynaptic secondary junctional folds changes following castration and testosterone replacement. Shown are transmission electron photomicrographs of cross-sections of neuromuscular junctions from normal (left), castrated (middle), and testosterone-treated (right) muscles. The small black granules are reaction product from the acetylcholinesterase stain (see Materials and Methods). The density of secondary junctional folds increases following castration, probably due to shrinkage of the muscle fiber membrane. Junctional fold density returns to normal levels following testosterone replacement. The density of folds from junctions from normal and manipulated muscles is summarized in Table 1 . Scale bar, $1 \mu \mathrm{m}$.

degree of remodeling at long time points seemed minor when compared to the extent of junctional enlargement observed with testosterone treatment. These results show that ongoing atrophy and hypertrophy of muscle fibers has a primary effect on junctional size rather than on the pattern or number of synaptic regions.

\section{Existing ACh receptor regions shrink and expand as muscle fibers atrophy and hypertrophy}

To study how junctional enlargement might be coupled to muscle fiber hypertrophy, and how this might be related to normal junctional growth during postnatal life, we followed the fate of
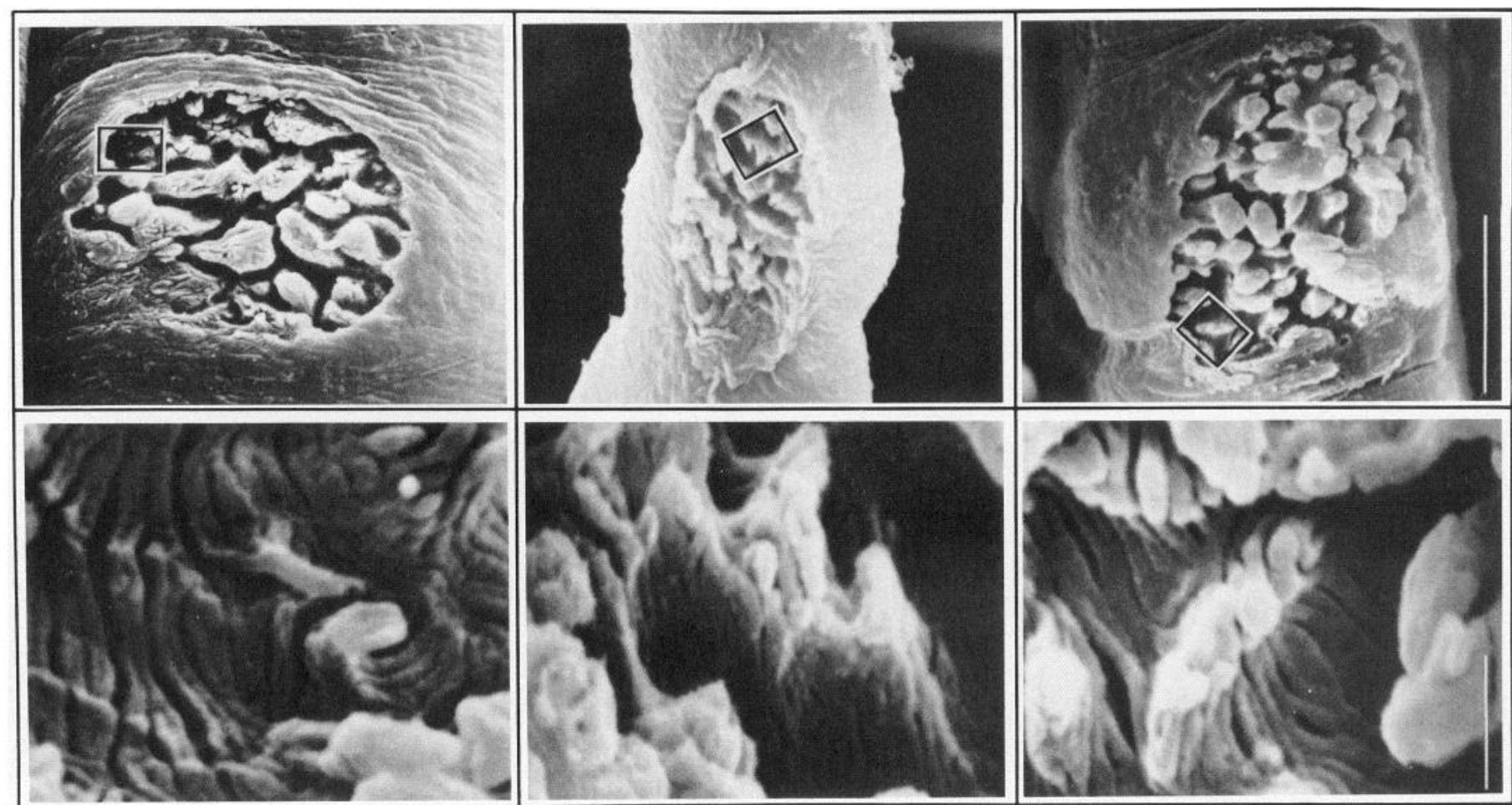

Normal

IMonth After Castration

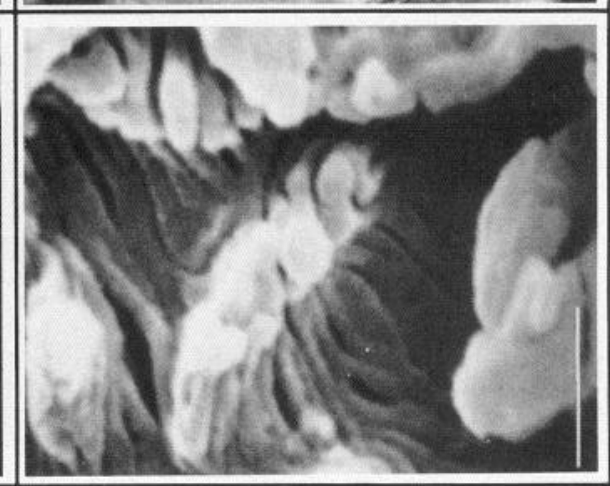

1 Month After Testosterone

Figure 8. Density of postsynaptic secondary junctional folds changes in parallel with junctional and muscle fiber size. Top, Scanning electron photomicrographs of the postsynaptic surface of bulbocavernosus neuromuscular junctions from normal (left), castrated (middle), and testosteronetreated (right) muscles. Scale bar, $10 \mu \mathrm{m}$. Bottom, Higher magnification view of boxed regions of junctions in top row. Junctional fold density increases following castration (Table 1). By multiplying the decrease in length or width of junctions from muscles 1 month after castration by the number of folds per linear micron, the estimate of the resulting total number of folds per junction is similar to that from normal muscles. Following testosterone replacement, junctional fold density returns to normal and the number of folds per junction is unchanged. The density and number of junctional folds for normal and manipulated muscles is summarized in Table 1. Scale bar, $1 \mu \mathrm{m}$. 
postsynaptic ACh receptors (irreversibly labeled with fluorescently tagged $\alpha$-bungarotoxin) as muscle fibers enlarged following testosterone treatment. In this way, we studied if and how ACh receptor molecules were redistributed as junctional size was altered. In each of the 23 junctions studied ( 5 animals), existing receptors were first observed to occupy a smaller synaptic area following castration (Fig. 6, middle panels). This loss of area could mean that $A C h$ receptors were selectively lost from the ends and edges of receptor regions and that the remaining receptors underwent a complex redistribution to recreate a smaller version of the receptor regions as well as the spaces between them. A far more likely explanation for this decrease in area, however, is that the entire postsynaptic ACh receptorrich region compacted as the muscle fiber atrophied.

After testosterone treatment, previously labeled receptors were observed to redefine a larger synaptic area (Fig. 6, right panels). New receptors (labeled with a second color of $\alpha$-bungarotoxin) were observed to be intercalated throughout the enlarged postsynaptic region and not preferentially at the ends or edges of receptor regions (Fig. 6, right bottom panel). This observation indicates that as muscle fibers hypertrophy, existing ACh receptor regions expand as if they were stretched. Thus, at the light microscopic level, muscle fiber atrophy following castration and hypertrophy following testosterone treatment give rise to changes in the size of postsynaptic regions that seem to occur by the same mechanism.

\section{Postsynaptic secondary junctional folds shrink and expand as muscle fibers change size}

We examined the number and density of postsynaptic secondary junctional folds (which are invaginations of the muscle fiber membrane, at the crests of which ACh receptors are located) following castration and testosterone replacement. In this way, we could study at the ultrastructural level the changes in postsynaptic specializations that accompany the shrinkage and expansion of androgen-manipulated muscle fibers. Analysis of junctional fold density from transmission electron photomicrographs showed that fold density in junctions from castrated muscles was significantly higher than in junctions from normal muscles (Fig. 7 and Table 1). After testosterone replacement, the density of junctional folds returned to normal levels (Fig. 7 and Table 1). These observations are consistent with the idea that the muscle fiber membrane becomes more compact as muscle fibers atrophy and reexpands as muscle fibers hypertrophy.

Analysis of secondary junctional fold density from scanning electron photomicrographs showed similar changes (Fig. 8 and Table 1). Moreover, scanning electron microscopic views allowed us to obtain an index of the total number of folds per junction. When junctional length or width was multiplied by the number of folds per linear micron (see Materials and Methods), we found that this number was similar in junctions from normal, castrated, and testosterone-treated muscles (Table 1). These results suggest that the density, but not the total number, of folds per junction changes as a result of changing muscle fiber size.

\section{Discussion}

We have studied changes in neuromuscular junction size and branching pattern as a result of manipulating muscle fiber size with androgens in the bulbocavernosus muscle of mice. Neuromuscular junctions from normal adult bulbocavernosus mus- cles grow but are otherwisc stably maintaincd. By altcring muscle fiber size with the removal and replacement of testosterone, our results show that junctional size is directly coupled to muscle fiber size without a change in the number or pattern of synaptic regions.

\section{Neuromuscular synapses grow without continual remodeling}

As previously observed in the mouse sternomastoid muscle (Balice-Gordon and Lichtman, 1990), bulbocavernosus neuromuscular junctions grow without a change in the pattern or number of pre- and postsynaptic regions. Thus, it is likely that this is the general means by which junctions grow in normal skeletal muscles. This lack of remodeling during growth should not, however, be taken as evidence that mammalian neuromuscular junctions cannot remodel, because in a number of normal and experimental situations, neuromuscular synapses do remodel extensively. For example, during the first weeks of postnatal life, both motor nerve terminal branches and the $\mathrm{ACh}$ receptor regions beneath them are eliminaled as multiple innervation regresses and the adult pattern of single innervation is established (Balice-Gordon and Lichtman, 1989). Similarly, following nerve crush and reinnervation of adult junctions, terminal and receptor regions are lost as transient terminal sprouts regress (Rich and Lichtman, 1989a). In adult slow muscles, loss of synaptic regions has also been reported (Wigston, 1989). Furthermore, when presynaptic motor nerve terminals are poisoned with botulinum toxin (Reiness and Lichtman, 1989), or when muscle fibcrs are damaged and allowed to regenerate (Rich and Lichtman, 1989b), there is a prolonged period of remodeling both pre- and postsynaptically. Finally, as animals mature into old age, neuromuscular junctions that were stable for long intervals begin to lose large synaptic regions and add new ones (Balice-Gordon and Lichtman, 1990). It appears, however, that growing junctions beyond the neonatal period have stable configurations in which changes in the number or pattern of synaptic regions are rare (see also Wigston, 1988).

Androgens modulate bulbocavernosus rnuscle fiber size directly

In order to understand the cellular mechanisms underlying junclional size changes as muscle fiber size is manipulated, it is necessary to know whether androgens affect muscle fibers, motor neurons, or both, because each has been shown to possess androgen receptors (Dube et al., 1976; Breedlove and Arnold, 1980). For example, testosterone might affect the activity of bulbocavernosus motor neurons, which in turn might have an effect on the size of their motor nerve terminals. Alternatively, testosterone might affect both motor neurons and muscle fibers in a highly coordinated fashion, which would lead to similar changes in the size of motor nerve terminals and muscle fibers. Finally, testosterone might act directly on bulbocavernosus muscle fibers and modulate their size primarily through that route.

One line of evidence strongly suggests that testosterone does, in fact, act directly on bulbocavernosus muscle fibers rather than on motor neurons to regulate muscle fiber size. Testosterone can spare androgen-sensitive muscles such as the bulbocavernosus from atrophy during development even in the complete absence of innervation (Fishman and Breedlove, 1989). Other experiments support the idea that androgens exert their anabolic effect directly on muscle fibers (Powers and Florini, 1975; Rand and Breedlove, 1987), and receptors that bind androgens have been shown to be present in large numbers in the bulbocaver- 
nosus muscle (Jung and Baulieu, 1972; Krieg et al., 1974; Dube et al., 1976).

Levels of testosterone seem to affect muscle fiber size by regulating the number of myofibrils. Myofibrils are lost in response to castration (Venable, 1966b), resulting in a decrease in intracellular volume, leading to a loss of area in the muscle fiber membrane. Subsequent testosterone treatment results in the addition of new myofibrils to muscle fibers (Venable, 1966b), which in turn results in expansion of the muscle fiber membrane.

\section{Postsynaptic specializations change size as a direct consequence of changes in the area of the muscle fiber membrane}

An obvious question is how muscle fiber size exerts an effect on the size of neuromuscular junctions. One possibility, supported by several observations in both normal and androgenmanipulated muscles, is that the size of postsynaptic specializations is directly coupled to the surface area of the postsynaptic muscle fiber membrane. Evidence for such coupling comes from the parallel changes in the length and width of bulbocavernosus neuromuscular junctions and muscle fibers during normal growth (see also Balice-Gordon and Lichtman, 1990). Furthermore, this correlation is maintained as androgen levels are altered, affecting muscle fiber size reversibly: junctional size is altered at the same time and to a similar extent as muscle fiber size. A direct link between the area of the muscle fiber membrane and junctional area is also supported by following the movements of labeled ACh receptors as muscle fibers changed size. As muscle fibers atrophy following castration, existing receptors were observed to define a smaller synaptic region. Not only did all of the receptor-rich regions become smaller, but so did the spaces between the regions, arguing that the entire region at the junction (both synaptic and extrasynaptic) decreased in area. As muscle fibers hypertrophy following testosterone replacement, existing $\mathrm{ACh}$ receptors redefined a larger synaptic area with increased spaces between receptor regions. These results are similar to the spreading apart of $\mathrm{ACh}$ receptor molecules into progressively larger areas during normal growth of neuromuscular junctions in the sternomastoid muscle (as well as in the bulbocavcrnosus). Furthermore, this expansion of receptor regions and the spaces between them can be mimicked acutely by stretching muscle fibers (Balice-Gordon and Lichtman, 1990). These observations suggest that the entire postsynaptic area shrinks and expands as a direct mechanical consequence of shrinkage or expansion of the muscle fiber membrane.

There are at least 3 ways postsynaptic specializations of the muscle fiber membrane might change ultrastructurally as junctions shrink and expand. One possibility is that the density of postsynaptic secondary junctional folds might increase as muscle fibers atrophy and return to normal as muscle fibers hypertrophy. Alternatively, if junctions decreased in size solely by a decrease in the size of the muscle fiber regions between synaptic regions, then one would expect that the density of postsynaptic folds might not be altered (Hanzlikova and Gutmann, 1978). This possibility is unlikely because the present results showed that the dimensions of synaptic regions changed to a similar degree as nonsynaptic regions. Finally, the total number of folds might decline as junctions shrink, and increase as junctions expand, if postsynaptic specializations were lost and added as muscle fibers changed size (Tobin and Pécot-Dechavassine, 1982).

In the present experiments, postsynaptic secondary junctional folds were observed to become more densely packed as muscle fibers atrophied following castration and less densely packed as muscle fibers hypertrophied following testosterone treatment. This change in density occurred without a change in the total number of folds per junction. These light and electron microscopic results suggest that the primary determinant of junctional size is the size of the postsynaptic muscle fiber as manifested by an effect on the surface area of the muscle fiber membranc.

Motor nerve terminals may change size as a direct consequence of adhesion to postsynaptic specializations

Because it seems likely that the size of postsynaptic specializations is directly determined by the surface area of the muscle fiber membrane, an issue of interest is the way motor nerve terminals keep pace with these changes in the postsynaptic membrane. The present results, together with previous evidence, strongly suggest that motor nerve terminals may be pulled along as $\mathrm{ACh}$ receptor regions change size by virtue of adhesion between pre- and postsynaptic regions (Balice-Gordon and Lichtman, 1990).

These results argue for a direct mechanical explanation for synaptic growth mediated by adhesion, rather than by, for example, diffusible trophic factors acting at a distance, which traditionally have bcen invoked to cxplain the matching of presynaptic terminals to the size of target cells and their precise alignment with postsynaptic specializations. While our results suggest that diffusible factors, trophic or otherwise, are unlikely to regulate coordinated pre- and postsynaptic growth in muscle, these results do not rule out a possible role for such factors in affecting nerve terminals in other circumstances (i.e., sprouting).

A passive mechanical relationship mediated by adhesion may underlie the "size matching" of neuromuscular junctions and muscle fibers not only during normal growth, but also in muscles that change size seasonally (Breedlove, 1986; Forger and Breedlove, 1987). Because pre- and postsynaptic elements of synapses are adherent throughout the nervous system, it is possible that adhesion also helps match synaptic size with postsynaptic target cell size and maintain synaptic connections in other parts of the nervous system.

\section{References}

Balice-Gordon RJ, Lichtman JW (1989) Competing motor nerve terminals and the ACh receptors underlying them are rearranged during synapse elimination. Soc Neurosci Abstr 15:565.

Balice-Gordon RJ, Lichtman JW (1990) In vivo visualization of the growth of pre- and postsynaptic elements of neuromuscular junctions in the mouse. J Neurosci 10:894-908.

Barker D, Ip MC (1966) Sprouting and degeneration of mammalian motor axons in normal and de-afferented skeletal muscle. Proc Natl Acad Sci USA 163:538-554.

Bleisch WV, Harrelson A (1989) Androgens modulate endplate size and $\mathrm{ACh}$ receptor density at synapses in rat levator ani muscle. $\mathbf{J}$ Neurobiol 20:189-202.

Breedlove SM (1986) Cellular analyses of hormone influence on motoneuronal development and function. J Neurobiol 17:157-176.

Breedlove SM, Amold AP (1980) Hormone accumulation in a sexually dimorphic nucleus in the rat spinal cord. Science 210:564-566.

Breedlove SM, Balice-Gordon RJ, Lichtman JW (1988) Neuromuscular junctions shrink and expand as muscle fibers change size: studies in an androgen sensitive muscle. Soc Neurosi Abstr 14:1209.

Desaki J, Uehara J (1981) The overall morphology of neuromuscular junctions as revealed by scanning electron microscopy. $J$ Neurocytol 10:101-110.

Dube JY, Lesage R, Tremblay R (1976) Androgen and estrogen binding in rat skeletal and perineal muscles. Can J Biochem 54:50-55. 
Fishman RB, Breedlove SM (1988) Neonatal androgen maintains sexually dimorphic perineal muscles in the absence of innervation. Muscle Nerve 11:553-560.

Forger $N G$, Breedlove SM (1987) Seasonal variation in mammalian striated muscle mass and motoneuron morphology. J Neurobiol 18 : $155-165$.

Guth L, Samaha FJ (1970) Procedure for histochemical demonstration of actomyosin ATPase. Exp Neurol 28:365-367.

Hanzlikova V, Gutmann E (1978) Effect of castration and testosterone administration on the neuromuscular junction in the levator ani muscle of the rat. Cell Tiss Res 189:155-166.

Hart BL, Melese d'Hospital Y (1983) Penile mechanisms and the role of the striated penile muscles in penile reflexes. Physiol Behav 31 : 807-813.

Jung I, Baulieu E (1972) Testosterone cytosol "receptor" in the rat levator ani muscle. Nature New Biol 237:24-25.

Karnovsky MJ, Roots L (1964) A "direct coloring" thiocholine method for cholinesterase. J Histochem Cytochem 12:219-221.

Krieg M, Szalay R, Voigt KD (1974) Binding and metabolism of testosterone and of $5 \alpha$-dihydrotestosterone in bulbocavernosus/levator ani (BCLA) of male rats: in vivo and in vitro studies. J Steroid Biochem 5:453-459.

I ichtman JW (1988) Dynamic aspects of synaptic connections at the vertebrate neuromuscular junction. In: The making of the nervous system (Parnavelas JG, Stern CD, Stirling RV, eds), pp 340-355. New York: Oxford U.P.

Lichtman JW, Magrassi L, Purves D (1987) Visualization of neuromuscular junctions over periods of several months in living mice. $\mathrm{J}$ Neurosci 7:1215-1222.

Magrassi L, Purves D, Lichtman JW (1987) Fluorescent probes that stain living nerve terminals. J Neurosci 7:1207-1214.

Menniti FS, Baum MJ (1981) Differential effects of estrogen and androgen on locomotor activity induced in castrated male rats by amphetamine, a novel environment or apomorphine. Brain Res 216:89107.

Powers LM, Florini JR (1975) A direct effect of testosterone on muscle cells in tissue culture. Endocrinology 97:1043-1047.

Rand MN, Breedlove SM (1987) Local site of action for testosterone's anabolic effect on rat bulbocavernosus. Soc Neurosci Abstr 13:54.
Reiness CG, Lichtman JW (1989) Pre- and postsynaptic remodcling in adult mouse muscle induced by botulinum toxin. Soc Neurosci Abstr 14:1209.

Rich MM, Lichtman JW (1989a) In vivo visualization of pre- and postsynaptic changes during synapse elimination in reinnervated mouse muscle. J Neurosci 9:1781-1805.

Rich MM, Lichtman JW (1989b) Motor nerve terminal loss from degenerating muscle fibers. Neuron 3:677-688.

Rohhins N (1988) Plasticity at motor nerve terminals. In: Nerve-target cell trophic communication (Fernandez H, ed), pp 199-215. Boca Raton, FL: CRC.

Sachs BD (1982) Role of the rat's striated penile muscles in penile reflexes, copulation and the induction of pregnancy. $\mathbf{J}$ Reprod Fertil $66: 433-443$.

Smith ER, Damassa DA, Davidson JM (1977) Hormone administration: peripheral and intracranial implants. In: Methods in psychobiology, Vol III (Meyers RD, ed), pp 259-279. New York: Academic.

lobin C, Pécot-Dechavassine M (1982) Effect of castration on the morphology of the motor end-plates of the rat levator ani muscle. Eur J Cell Biol 26:284-288.

Venable JH (1966a) Constant cell populations in normal, testosteronedeprived and testosterone-stimulated levator ani muscles. Am J Anat 119:263-270.

Venable JH (1966b) Morphology of the cells of normal, testosteronedeprived and testosterone-stimulated levator ani muscles. Am J Anat 119:271-302.

Wainman P, Shipounoff GC (1941) The effects of castration and testosterone propionate on the striated perineal musculature in the rat. Endocrinology 29:975-978.

Wernig A, Herrera AA (1986) Sprouting and remodelling at the nervemuscle junction. Prog Neurobiol 27:251-291.

Wigston DJ (1990) Repeated in vivo visualization of neuromuscular junctions in adult mouse lateral gastrocnemius. J Neurosci 10:17531761.

Wigston DJ (1989) Remodeling of neuromuscular junctions in adult mouse soleus. J Neurosci 9:639-647. 